\title{
Rap1, a Small GTP-Binding Protein Is Upregulated During Arrest of Proliferation in Human Keratinocytes
}

\author{
N.J. D'SILVA, ${ }^{1}$ * R.S. MITRA, ${ }^{1}$ Z. ZHANG, ${ }^{1}$ D.M. KURNIT, ${ }^{2}$ C.R. BABCOCK, ${ }^{1}$ P.J. POLVERINI, ${ }^{1}$ \\ AND T.E. CAREY ${ }^{3}$ \\ ${ }^{1}$ Department of Oral Medicine, Pathology and Oncology, \\ University of Michigan School of Dentistry, Ann Arbor, Michigan \\ ${ }^{2}$ Departments of Pediatrics and Human Genetics, \\ University of Michigan Medical Center, Ann Arbor, Michigan \\ ${ }^{3}$ Department of Otolaryngology, Laboratory of Head and Neck Cancer Biology, \\ The University of Michigan Medical School and the Comprehensive Cancer Center, \\ Ann Arbor, Michigan
}

\begin{abstract}
Rap1 is a small GTP-binding protein (SMG) that exists in two 95\% homologous isoforms, rap1 A and rap1B. The functions of the rap1 proteins are not well understood. In this report we examined expression and function of rap1 in primary (HOKs) and immortalized (IHOKs) human oral keratinocytes under different growth conditions. In HOKs, rap1 increased with passage number, suggesting a role in differentiation and arrest of proliferation. Similarly, when inhibition of proliferation and differentiation were induced in $\mathrm{HOKs}$ by $1.2 \mathrm{mM} \mathrm{CaCl}_{2}$, both rap1 and involucrin increased with increasing concentrations of $\mathrm{CaCl}_{2}$. However, when similar experiments were done with IHOKs, which continue to proliferate in the presence of $1.2 \mathrm{mM} \mathrm{CaCl}_{2}$, the increase in involucrin expression was similar to HOKs but there was no substantial increase in rap1, suggesting that increased expression of rap 1 is linked to inhibition of proliferation rather than differentiation of keratinocytes. Upon transfection of immortalized keratinocytes with rapGAP, which inactivates both isoforms of endogenous active rap1, enhanced proliferation was observed. Thus, we conclude that rap1 inhibits proliferation in keratinocytes. J. Cell. Physiol. 196: 532-540, 2003. @ 2003 Wiley-Liss, Inc.
\end{abstract}

Small GTP-binding proteins (SMGs) are divided into five subfamilies, namely ras, rho, rab, arf, and ran (Vojtek and Der, 1998). Members of these subfamilies regulate their functions by shuttling between inactive GDP- and active GTP-bound states. In general, ras subfamily proteins are key players in receptor-linked signaling pathways that control cell growth and differentiation (Bokoch, 1993; Wagner and Williams, 1994). Ras and rap1 are members of the ras subfamily. Growth factor-induced ras-activation triggers the mitogen activated protein kinase (MAPK) signaling cascade, which culminates in gene transcription, cell proliferation or differentiation (Herrmann and Nassar, 1996).

Persistent up-regulation of the ras-signaling pathway facilitates uncontrolled cell proliferation and malignant transformation via the MAPK pathway. Ras is one of the most frequently mutated oncogenes in cancer (Barbacid, 1987; Grunicke and Maly, 1993) but ras mutations are not always detected in tumor cells with an aberrantly upregulated MAPK pathway (Vojtek and Der, 1998). Of particular interest is that ras mutations have been identified in only $5 \%$ of oral squamous cell carcinomas occurring in patients in the Western hemisphere, in contrast to $35 \%$ in India and Southeast Asia where betel nut chewing and reverse smoking are prevalent (Saranath et al., 1991; Clark et al., 1993; Jordan and Daley, 1997).
Mutations or activation of other ras-like proteins may play a role in oral carcinogenesis because SMGs are integral members of the growth cycle (Bokoch, 1993). Rap1 is a ras-like protein that appears to have a role in cell proliferation (Kitayama et al., 1989; Yoshida et al.,

Abbreviations: SMG, small GTP-binding protein; HOK, primary human oral keratinocytes; IHOK or HOK16B, immortalized human oral keratinocytes; TBS, tris buffered saline; RT-PCR, reverse transcriptase-polymerase chain reaction; MAPK, mitogen activated protein kinase; GAPDH, glyceraldehyde-3-phosphate dehydrogenase.

Contract grant sponsor: Office of the Vice President for Research of the University of Michigan (to N.J.D.); Contract grant sponsor: NIH-NIDCR (to N.J.D.); Contract grant number: DE00452-01.

P.J. Polverini's present address is University of Minnesota School of Dentistry, Minneapolis, MN 55455.

*Correspondence to: N.J. D'Silva, Department of Oral Medicine, Pathology and Oncology, University of Michigan, School of Dentistry, 1011 N. University Ave, Rm 5217, Ann Arbor, MI 48109-1078. E-mail: njdsilva@umich.edu

Received 3 January 2003; Accepted 28 March 2003

DOI: $10.1002 /$ jcp. 10331 
1992; Altschuler and Ribeiro-Neto, 1998). It is a ubiquitously expressed protein that has two isoforms, rap1A and rap1B, products of different genes located on chromosomes 1 and 12, respectively (Noda, 1993). Rap1A, also called smg p21 or Krev1, is $95 \%$ homologous to rap1B, from which it differs by only 9 amino acids (of a total of 184). Six of these are located in the C-terminus between amino acid residues 171-184 (Bokoch, 1993).

Rap1 is closely homologous to ras in the ras transformation domains, which include the GTP-binding region, effector domain, and membrane attachment site (Farrell et al., 1992). The identical effector domains of ras and rap1 suggested an antagonistic or complementary role in the same signaling pathway (Altschuler et al., 1995). In fact, rap1A was originally identified as a rastumor suppressor gene in ras transformed NIH 3T3 fibroblasts (Kitayama et al., 1989). Rap1A was postulated to function by competitively inhibiting ras GTPase activating protein (rasGAP), a ras effector (Kitayama et al., 1989). Furthermore, rap1A was also shown to facilitate cell proliferation by inducing DNA synthesis when microinjected into Swiss 3T3 cells (Yoshida et al., 1992). Rap1B, the other rap1 isoform, has been reported to induce proliferation and morphologic transformation of Swiss 3T3 cells (Altschuler and Ribeiro-Neto, 1998). In neuronal cells, rap1B acts independently of ras, via the MAPK cascade to promote differentiation (Vossler et al., 1997). Thus, there is confusion regarding the functions of the rap1 isoforms.

Due to the diversity of opinions regarding the functions of rap1A and rap1B, it is important to investigate the functions of these proteins in the context of a specific cell type. Although the majority of tumors are epithelial in origin $(>80 \%)$, the role of rap1 in growth and differentiation of epithelial cells remains relatively unexplored. In the present study, we investigated the expression and function of $\operatorname{rap} 1 \mathrm{~A}$ and rap1B in human oral keratinocytes and show that active rap1-GTP is upregulated in differentiating keratinocytes and that it inhibits proliferation. Hence, rap1 may have a tumor suppressor role in oral epithelium.

\section{MATERIALS AND METHODS Cell culture}

Primary oral keratinocytes were cultured from human gingival tissue as described previously (Oda and Watson, 1990). Discarded gingival tissue from third molar extraction sites or from periodontal surgery, was collected (after appropriate Institutional Review Board for Human Subject Research approval) in PBS with antibiotic/antimycotic (penicillin $200 \mathrm{U} / \mathrm{ml}+$ streptomycin $200 \mu \mathrm{g} / \mathrm{ml}+$ amphotericin B $50 \mu \mathrm{g} / \mathrm{ml}$; GIBCO, Carlsbad, CA), washed extensively in the same media, and incubated overnight in $4 \mathrm{mg} / \mathrm{ml}$ Dispase (Collaborative Biomedical/Becton Dickinson, Bedford, MA). The epithelium was dissociated from the connective tissue, trypsinized and dispersed into single cells and seeded and grown in low-Ca ${ }^{2+}(0.03 \mathrm{mM} \mathrm{CaCl})$, serum-free Keratinocyte Growth Medium (KGM, Biowhittaker/ Clonetics Corp., Walkersville, MD). This was designated P1 (passage 1). Keratinocytes were routinely passaged using $0.05 \%$ trypsin/0.53 mM EDTA. Keratinocytes between passage numbers 1 and 4 were used. An HPV16 immortalized human oral keratinocyte cell line
(HOK 16B, gift from Dr. No-Hee Park, University of California, Los Angeles) was maintained in low-Ca ${ }^{2+}$ KGM (Park et al., 1991).

\section{Treatment of cells with high $\mathrm{Ca}^{2+}$}

To analyze calcium-induced changes in keratinocytes, cells were cultured for 3 or 7 days in KGM supplemented with $1.2 \mathrm{mM} \mathrm{CaCl}_{2}$

\section{Proliferation assay}

Keratinocyte proliferation was measured by seeding approximately 15,000-20,000 cells per well in a 24-well culture plate in KGM. One day later, the cells were washed and incubated in KGM or KGM supplemented with $1.2 \mathrm{mM} \mathrm{CaCl}_{2}$ (day 0). Media was changed on $\mathrm{d} 0$, $\mathrm{d} 2, \mathrm{~d} 4$, and $\mathrm{d} 6$. Cells were counted on $\mathrm{d} 0, \mathrm{~d} 1, \mathrm{~d} 3, \mathrm{~d} 5$ and $\mathrm{d} 7$. After the appropriate incubation period, the wells were washed once with PBS and the cells were harvested with trypsin/EDTA and counted with a hemocytometer. Cell viability was assessed on the basis of Trypan blue exclusion. Values represent means and standard deviation based on three wells per data point in a single experiment. The experiment was repeated at least three times with similar results.

\section{Western blot analysis}

For the preparation of lysates, keratinocytes were washed once with ice-cold PBS and lysed in 1\% NonidetP40 lysis buffer containing protease inhibitors on ice for $15 \mathrm{~min}$. The particulate material was pelleted by centrifugation at $15,700 \mathrm{~g}$ for $10 \mathrm{~min}$ at $4^{\circ} \mathrm{C}$. The supernatant was collected and protein content was measured by the Bio-Rad protein assay (Bio-Rad, Richmond, CA). Equal amounts of protein $(25-50 \mu \mathrm{g})$ or equivalent cell numbers, were electrophoresed on SDS/12\% polyacrylamide gels and transferred to nitrocellulose membranes (Schleicher and Schuell, Keene, NH). Prior to blocking, the membrane was stained with Ponceau S for verification of equivalency of protein loading and efficient transfer. The membranes were incubated in 5\% non-fat dry milk in Tris-buffered saline (TBS) for $1 \mathrm{~h}$ at room temperature (RT) to block non-specific binding. Membranes were placed in the primary antibody for $1 \mathrm{~h}$ at RT or overnight at $4{ }^{\circ} \mathrm{C}$. Primary antibody concentrations were: rabbit anti-rap1 affinity purified polyclonal antibody (1:150, Santa Cruz, CA); mouse anti-involucrin AB-1 (Sy5) monoclonal antibody (1:1000; Neomarkers, Fremont, CA); or mouse antiGAPDH (1:5000; Chemicon International, Temecula, CA). Membranes were washed in TBS containing $0.1 \%$ Tween-20 (TBS-T) (Bio-Rad). Affinity-purified horseradish peroxidase-linked donkey anti-rabbit or goat anti-mouse IgG (Jackson ImmunoResearch Laboratories, West Grove, PA) as a secondary antibody were used to detect primary antibodies. Visualization of the immunoreactive proteins was accomplished by the chemiluminescence system (Amersham, Arlington Heights, IL or Pierce, Rockford, IL) and exposure to film.

\section{Cell immunostaining}

Cultured HOKs were fixed in acetone and methanol, air dried, and stained for rap1 and involucrin as follows. Slides were incubated with rabbit anti-rap1, mouse antiinvolucrin, rabbit IgG or mouse IgG, for $1 \mathrm{~h}$ at RT. For 
double immunofluorescence labeling, the slides were initially treated with rabbit anti-rap1 polyclonal antibody or rabbit IgG followed by immunodetection using FITC (fluorescein isothiocyanate)-conjugated donkey anti-rabbit IgG (Jackson Immunoresearch). The washed slide was subsequently stained with mouse anti-involucrin or mouse IgG, washed with PBS, and then exposed to Texas red conjugated goat anti-mouse IgG (Jackson Immunoresearch). The cells were visualized on a laser scanning confocal microscope (MRC600, Bio-Rad). Slides were photographed with a $63 \times$ oil immersion lens, digitized and processed with Adobe Photoshop software (ver 5.0, San Jose, CA).

\section{$\left[\alpha_{-}^{-32} \mathbf{P}\right]$ GTP-blot overlay assay}

Low molecular mass GTP-binding proteins were detected by the radiolabeled GTP-blot overlay method as described previously (D'Silva et al., 1997). Briefly, cell lysates and molecular weight standards were separated by SDS 12\% PAGE, transferred to nitrocellulose membranes and blocked with GTP-binding buffer $(0.3 \%$ Tween-20, $5 \mu \mathrm{M} \mathrm{MgCl} 2,1 \mathrm{mM}$ potassium phosphate, $100 \mu \mathrm{M}$ ATP, $50 \mathrm{mM}$ Tris-HCl, pH 7.5). Subsequently, the membranes were incubated in the same buffer containing $100 \mathrm{mM}$ DTT and $1 \mu \mathrm{Ci}\left[\alpha_{-}{ }^{32} \mathrm{P}\right]-\mathrm{GTP} / \mathrm{ml}$ (Dupont-New England Nuclear Research Products, Boston, MA), with or without unlabeled $100 \mu \mathrm{M}$ GTP for $60 \mathrm{~min}$ at RT. The membranes were washed in the same buffer without DTT, were air dried and exposed to autoradiographic film for $1 \mathrm{~h}$ to 7 day.

\section{Total RNA isolation}

RNA was isolated from cell pellets or cells in culture dishes using the Total RNAEasy kit (Qiagen, Bothell, WA) or TRIZOL (GIBCO), using the respective manufacturer's protocol.

\section{Real time reverse transcriptase polymerase chain reaction (Q-RT-PCR)}

Real time RT-PCR was performed using the PerkinElmer TaqMan system (PE Applied Biosystems, Foster City, CA) (Heid et al., 1996). Briefly, two sets of rap1 isoform-specific primers were designed in exons that spanned the intron-exon boundaries in the region corresponding to the $\mathrm{C}$-termini of $\operatorname{rap} 1 \mathrm{~A}$ and rap1B (Rap1A - forward primer: CGGGTTAAGGACACGGAAGA, reverse primer: TCTTTGCCAACTACTCGCTCATC, TaqMan probe: TGATTTTGGTTGGCAATAAATGTGACCTGG; Rap1B-forward primer: AACAGATTCTTCGAGTTAAAGACACTGA, reverse primer: TTTGACCTTGTTCCTTCCCTACA, TaqMan probe: TGTTCCAATGATTCTTGTTGGTAATAAGTGTGACTTG). The primers did not amplify genomic DNA and demonstrated isoform specificity when tested against plasmids containing either the rap1A or rap1B cDNA sequences (kind gift from Dr. Veronique Pizon, Institut Andre Lwoff, France). The TaqMan probes, which are designed to bind to the middle of the regions being amplified, have reporter and quencher fluorescent dye molecules attached to each probe. During the extension cycle, the reporter dye is cleaved by the nuclease component of the polymerase. This releases the reporter from the quencher, thereby allowing the reporter to fluoresce. This fluorescence is quantified and during the logarithmic phase of the reaction is proportional to the amount of RNA being amplified.

\section{Transfections}

pcDNA 3.1-rapGAP plasmid was a kind gift from Dr. P. Stork (Oregon Health Sciences University, OR). The pcDNA 3.1 empty vector was used as a control for transfection effects on endogenous gene expression. Transfection efficiency was assessed with the pcDNA 3.1- $\beta$-galactosidase plasmid and commercially available detection reagent (Boehringer, Mannheim, Germany) followed by enumeration of positive staining cells per hundred cells in 25 high power fields.

For proliferation assays on transfected keratinocytes, immortalized keratinocytes were seeded at $\sim 200,000$ cells/dish in $60 \mathrm{~mm}$ dishes. Two days later the cells were transfected with Effectene (Qiagen, CA) according to the manufacturer's instructions in DMEM/ FBS. Media was changed to KGM $24 \mathrm{~h}$ after transfection. The total number of cells was counted on the day of transfection and at $24 \mathrm{~h}$ intervals thereafter for 4 day as described above.

\section{Rap1 activation assay}

The rap-binding domain of ral-GDS binds only the active form of rap1 (Franke et al., 1997). The construct for ral-GDS was a generous gift from Dr. Johannes L. Bos (University Medical Centre Utrecht, The Netherlands). Glutathione S-transferase (GST) tagged ralGDS was purified from bacterial cells expressing the protein and used to pull-down the active form of rap1B, as described previously (Franke et al., 1997).

\section{Data analysis}

Molecular mass determination of SMGs in Figure 1, was done using Alphaease ${ }^{\mathrm{TM}}$ software, version 5.5 (Alpha Innotech Corporation, San Leandro, CA). Statistical analysis was done by a Student's $t$-test.

\section{RESULTS \\ Rap1 expression is upregulated in consecutive passages of keratinocytes}

Small GTP-binding proteins from the ras subfamily have been shown to play a role in proliferation or differentiation of cells. Hence, in initial experiments, rap1 expression was investigated in sequential passages of primary keratinocytes. Primary normal human oral keratinocytes (HOK) may be cultured in vitro for approximately $3-5$ passages before the cells no longer proliferate (Oda and Watson, 1990). This cessation of proliferation has been attributed to aging and/or differentiation (Norsgaard et al., 1996). Cell lysates were prepared from sequential passages of freshly isolated adult HOKs until the cells ceased to proliferate, usually about 3-4 passages in our experiments. Lysates were normalized to cell number and were assayed for the presence of SMGs, particularly rap1. When separated by gel electrophoresis and immobilized on a nitrocellulose membrane, at least three SMG proteins with molecular weights of $\sim 22,24$, and $27 \mathrm{kDa}$, were detected by GTP overlay (Fig. 1A). There was increased binding of $\left[\alpha{ }^{32} \mathrm{P}\right]-$ GTP in each sequential passage of these primary oral keratinocytes (Fig. 1A). On an exact replicate membrane, an excess of unlabeled GTP $(100 \mu \mathrm{M})$ 


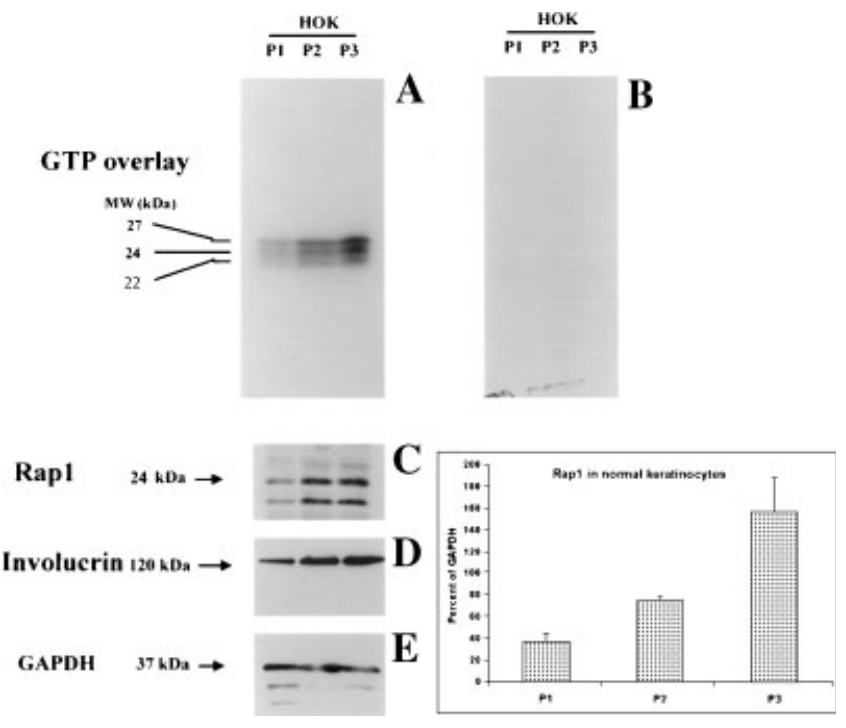

Fig. 1. Autoradiograph of GTP-binding and ECL detection of rap1, involucrin and GAPDH in cell lysates from sequential passages of primary human oral keratinocytes. HOKs, passages 1, 2 and 3 (P1, P2, P3; $8 \times 10^{4}$ cells/lane) were electrophoresed and transferred to membranes that were incubated with $\left[\alpha_{-}{ }^{32} \mathrm{P}\right]$ GTP in GTP-binding buffer with unlabelled $100 \mu \mathrm{M}$ ATP minus (A) or with unlabeled $100 \mu \mathrm{M}$ GTP (B). The membranes were exposed to film for $45 \mathrm{~min}$. The membrane that was previously incubated with cold GTP (B), was subsequently exposed to rap1 antibody (C), developed, stripped between successive antibodies, and successively tested with involucrin (D), or GAPDH (E) antibodies as indicated. The rap1 data (C) were normalized to GAPDH (E) and expressed as percent of GAPDH (F). The immunoblot data are representative of three independent experiments.

successfully competed for radiolabeled GTP thereby showing specificity of binding for these peptides (Fig. 1B). However, the presence of $100 \mu \mathrm{M}$ ATP in the binding buffer did not eliminate $\left[\alpha^{32} \mathrm{P}\right]-\mathrm{GTP}$ binding thereby confirming nucleotide specificity (Fig. 1A). Thus, at least three SMGs are upregulated in sequential passages of HOKs.

As shown in Figure $1 \mathrm{C}$ and $\mathrm{F}$, when the membrane from Figure 1B was probed with anti-rap1 antibody, rap1 was upregulated in the NP40-soluble fraction of sequential passages of HOKs. To evaluate whether this upregulation occurs concurrently with induction of differentiation, the same membrane was blotted again with a mouse anti-involucrin antibody. As shown in Figure 1D, involucrin was upregulated in sequential passages of HOKs. Equivalency of loading was assayed by blotting the same membrane a third time with mouse anti-GAPDH (glyceraldehyde-3-phosphate dehydrogenase) monoclonal antibody (Fig. 1E). Thus, rap1 is one of the SMGs upregulated in terminally differentiating $\mathrm{HOK}$ s.

\section{Both rap1 isoforms are expressed but neither is transcriptionally upregulated in terminally differentiated keratinocytes}

To verify which rap1 isoform is upregulated in sequential passages of HOKs, we performed real time RT-PCR. Both rap1A and rap1B mRNAs were present in sequential passages of primary HOKs (Fig. 2). In all passages, Rap1B mRNA was 10-fold higher than that of rap1A. No upregulation in either mRNA was noted between $\mathrm{P} 1$ and subsequent passages, suggesting that differences in protein expression may be translationally regulated.

\section{Rap1 actuvation is correlated with inhibition of proliferation}

Since terminal differentiation involves both growth inhibition and expression of differentiation markers, further studies were conducted to investigate the association between rap1 and these two processes in keratinocytes. For these studies we took advantage of the disparity in growth inhibitory effects induced by $\mathrm{CaCl}_{2}$ in primary and immortalized keratinocytes. Proliferation assays were performed on primary and immortalized keratinocytes in the presence of 0.03 and $1.2 \mathrm{mM} \mathrm{CaCl}_{2}$. In primary $\mathrm{HOKs}$, proliferation increased significantly at day 1 and started to plateau at day 3 , following addition of $1.2 \mathrm{mM} \mathrm{CaCl}_{2}$ (Fig. 3A). By day 7 , the total cell number plateaued in the cells grown in $0.03 \mathrm{mM} \mathrm{CaCl}_{2}$ and decreased in the cells grown in $1.2 \mathrm{mM} \mathrm{CaCl}_{2}$. In contrast to primary $\mathrm{HOKs}$, the IHOKs showed no $\mathrm{CaCl}_{2}$-induced $(1.2 \mathrm{mM})$ increase in proliferation at day 1 (Fig. 3B). Instead the cell number increased in both control $(0.03 \mathrm{mM})$ and $1.2 \mathrm{mM} \mathrm{CaCl}_{2^{-}}$ treated samples linearly through day 7 (Fig. 3B). Thus, growth inhibition occurred in primary keratinocytes but not in immortalized keratinocytes upon prolonged treatment with $\mathrm{CaCl}_{2}$. In contrast, differentiation, the other criterion for terminal differentiation, occurred in both primary and immortalized keratinocytes upon treatment with $1.2 \mathrm{mM} \mathrm{CaCl} \mathrm{Cl}_{2}$. Expression of the differentiation marker, involucrin, was induced at day 1 and was further increased by day 7 with $1.2 \mathrm{mM} \mathrm{CaCl}_{2}$ in primary keratinocytes (Fig. 3C). In immortalized keratinocytes, involucrin expression was highly upregulated at day 7 (Fig. 3E). Equivalency of loading was assayed by blotting the same membrane with mouse anti-GAPDH antibody (Fig. 3D and F, respectively). Thus, in normal primary keratinocytes, $1.2 \mathrm{mM} \mathrm{CaCl}_{2}$ triggered both

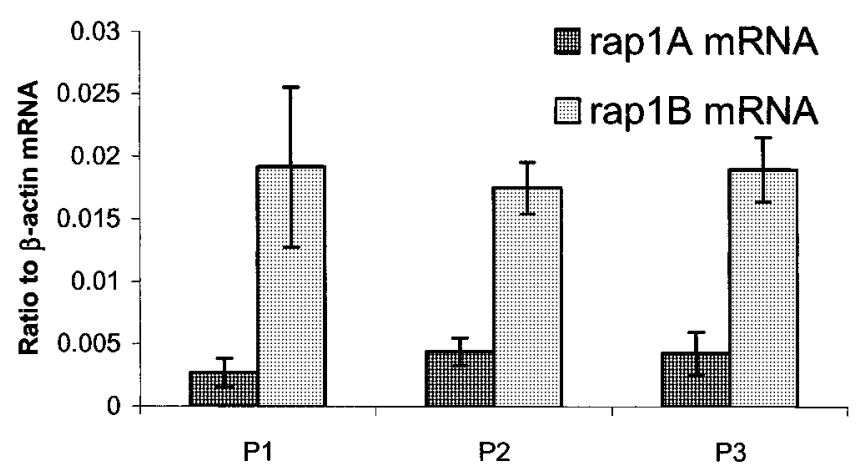

Fig. 2. Quantification of rap1A and rap1B mRNAs in sequential passages of primary human oral keratinocytes. Total RNA was prepared and real time RT-PCR was performed using the PerkinElmer TaqMan system as described in the "Materials and Methods." Rap1A and rap1B are normalized to $\beta$-actin. The data are the mean \pm SEM of four replicates in each of at least two independent experiments. The data are representative of three independent primary keratinocyte cell cultures. 


\section{Primary Keratinocytes}
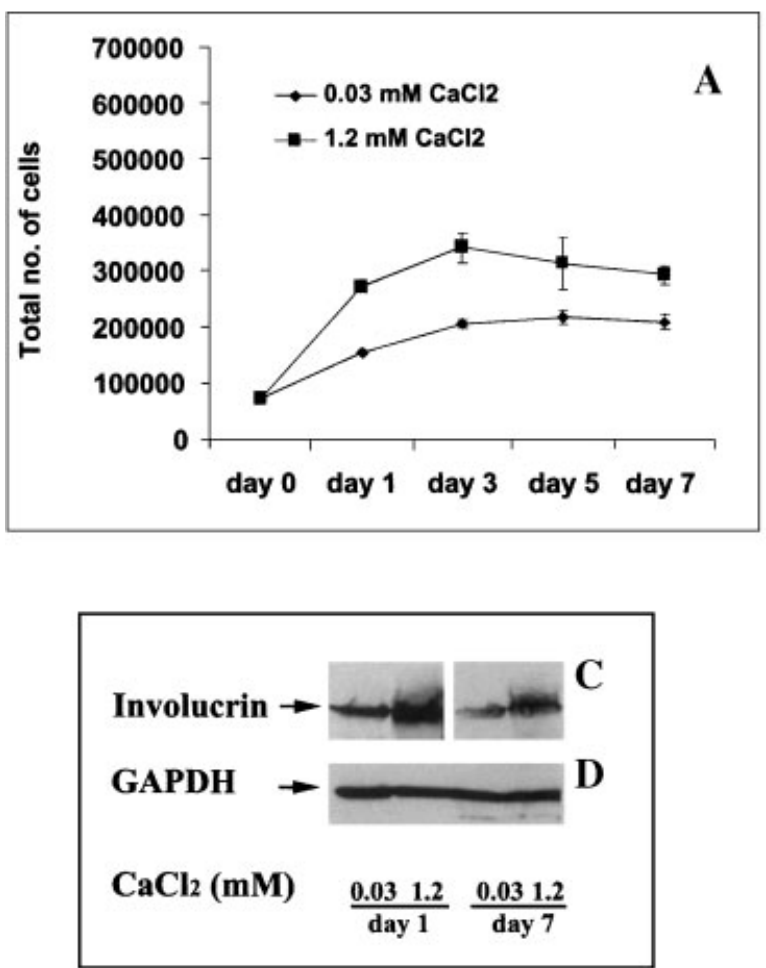

Fig. 3. $\mathrm{CaCl}_{2}$-induced proliferation and differentiation of primary and immortalized HOKs. Primary (A) or immortalized human oral keratinocytes $(\mathbf{B})$ were treated with 0.03 or $1.2 \mathrm{mM} \mathrm{CaCl}_{2}$ for 7 days and proliferation was measured as described in "Materials and Methods." The values represent means of cell number \pm SD (in A and B) based on 3 wells/data point in a single experiment. The experiment is representative of three experiments with similar results. Lysates from 0.03 or $1.2 \mathrm{mM} \mathrm{CaCl}_{2}$-treated primary or

differentiation and cessation of proliferation, whereas in immortalized keratinocytes, differentiation, but not growth inhibition, was observed.

Taking advantage of this system, cell lysates were prepared after treatment of primary and immortalized

\section{Primary keratinocytes}

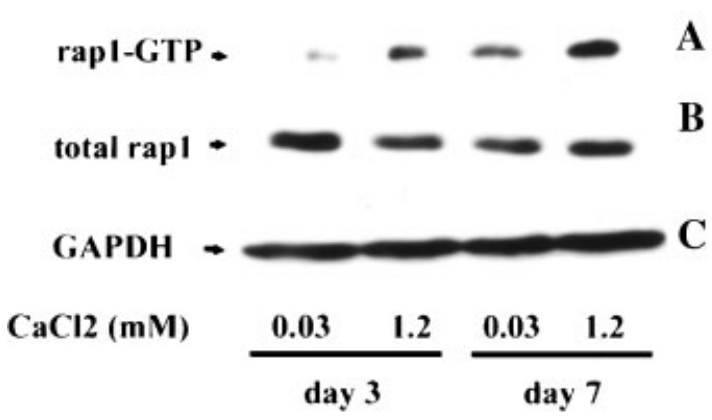

Fig. 4. Immunochemiluminescent detection of active GTP-bound rap1, total rap1 and GAPDH in cell lysates from primary and immortalized human oral keratinocytes treated with 0.03 and $1.2 \mathrm{mM} \mathrm{CaCl}_{2}$. Active GTP-bound rap1 was retrieved from lysates of primary $(400 \mu \mathrm{g})$ or immortalized $(600 \mu \mathrm{g})$ keratinocytes using the rap1 activation assay as described in "Materials and Methods." The retrieved active protein from the different treatment groups was

\section{Immortalized keratinocytes}
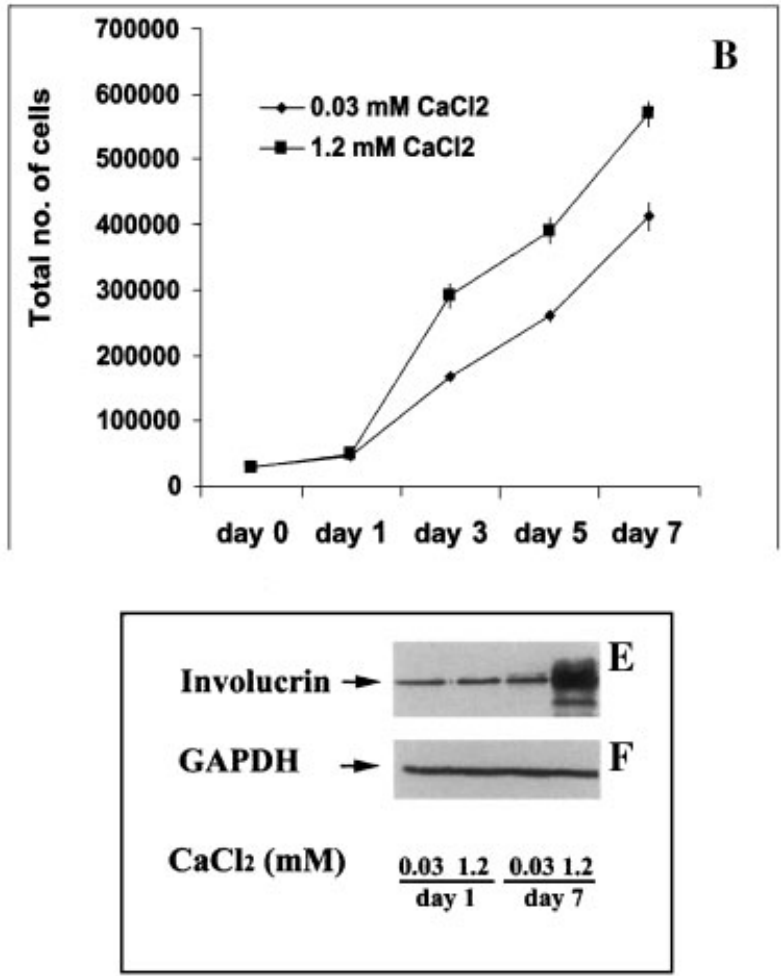

immortalized keratinocytes, were electrophoresed and transferred to membranes which were exposed to involucrin (C and $\mathbf{E}$, respectively) or GAPDH (D and F, respectively) antibodies. For primary keratinocytes, the involucrin signals at 7 day were much stronger than the 1 day signals. Therefore, chemiluminographs of 7-day samples were obtained after overnight decay of the substrate. The data are representative of two independent experiments.

HOKs with 0.03 or $1.2 \mathrm{mM} \mathrm{CaCl}_{2}$ for 3 or 7 days. In primary keratinocytes rap1 activation, as assayed by an increase in active GTP-bound rap1, increased from day 3 to day 7 in primary keratinocytes (Fig. 4A) in both 0.03 and $1.2 \mathrm{mM} \mathrm{CaCl}{ }_{2}$-treated cells. In contrast, immorta-

\section{Immortalized keratinocytes}

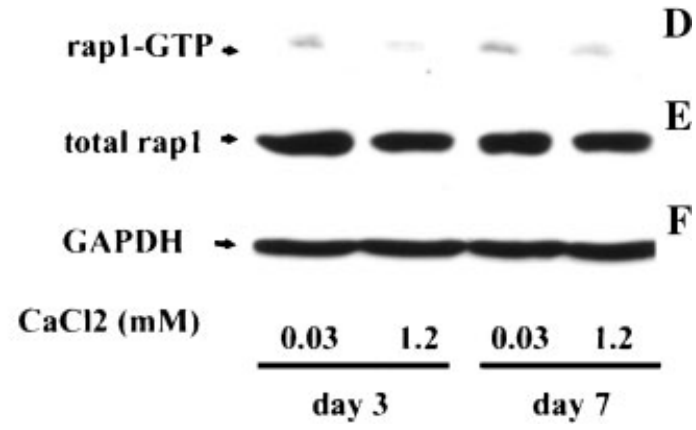

electrophoresed, transferred to membranes and exposed to rap1 antibody (A and $\mathbf{D}$ ). To normalize the data to total rap1 and GAPDH, corresponding cell lysates $(20 \mu \mathrm{g})$ were electrophoresed and transferred to membranes, which were exposed to $\operatorname{rap} 1(\mathbf{B}$ and $\mathbf{E})$, or GAPDH $(\mathbf{C}$ and $\mathbf{F})$ antibodies. The data are representative of three independent experiments. 
lized keratinocytes, which exhibit no growth inhibition, exhibit minimal rap1 activation in both 0.03 and $1.2 \mathrm{mM}$ $\mathrm{CaCl}_{2}$-treated cells at day 3 and 7 (Fig. 4D). In both primary and immortalized keratinocytes, there was no significant change in total rap1 expression (Fig. 4B and $\mathrm{E}$, respectively) at equivalent protein loading, as assessed by GAPDH (Fig. $4 \mathrm{C}$ and $\mathrm{F}$, respectively), at both days 3 and 7 .

\section{Rap1 does not coexpress with involucrin, a differentiation marker}

To verify the lack of association between rap1 and differentiation, concurrent expression of rap1 and involucrin was investigated by immunofluorescence double labeling. Rap1 was detected in HOKs cultured on Lab-Tek slides by FITC-labeled rabbit anti-rap1 polyclonal antibody, and involucrin was detected by Texas Red-labeled mouse anti-involucrin antibody and visualized by confocal microscopy (Fig. 5). These cell cultures included both small undifferentiated and large differentiated keratinocytes. Rap1 is strongly expressed in the small, undifferentiated keratinocytes (Fig. 5A,B, arrowheads). In contrast, the larger keratinocytes exhibit strong expression of involucrin, a marker of differentiation in HOKs, without a corresponding increase in rap1 (Fig. 5A,B, arrows) (Dotto, 1999). In Figure 5C and $\mathrm{D}$, the rap1 and involucrin antibodies are shown alone respectively with non-immune mouse IgG (Fig. 5C) and non-immune rabbit IgG (Fig. 5D), the primary antibody controls for involucrin and rap1, respectively. The nonimmune IgGs were appropriately negative thereby indicating specificity of staining. Thus it can be seen in this figure that some involucrin positive cells do not strongly express rap1, suggesting that rap1 upregulation and differentiation in keratinocytes are distinct and separate processes.

\section{RapGAP increases growth in immortalized keratinocytes}

Based on the preceding observations, we postulated that rapGAP (rap-GTPase activating protein), a protein that inactivates rap1-GTP, can enhance proliferation in immortalized keratinocytes. As assessed by $\beta$-galactosidase staining after $24 \mathrm{~h}$, we obtained a transfection efficiency of $\sim 10 \%$ with pcDNA 3.1- $\beta$-gal (Fig. 6A), similar to that reported previously (Zellmer et al., 2001). Immortalized keratinocytes were transfected with pcDNA 3.1-rapGAP and assessed for effects on proliferation. RapGAP expression in transfected keratinocytes was confirmed by immunoblot analysis (Fig. 6B) which showed strong expression in pcDNA 3.1-rapGAP transfected immortalized keratinocytes compared to the control vector transfected cells. The total number of cells and cell viability were assessed by trypan blue enumeration assay. As shown in Figure 6C, an increase in the total number of cells was observed in keratinocytes transfected with rapGAP. By day 4 , this increase was significantly different $(P<0.005)$ from the control vector

\section{Rap1 + Involucrin}

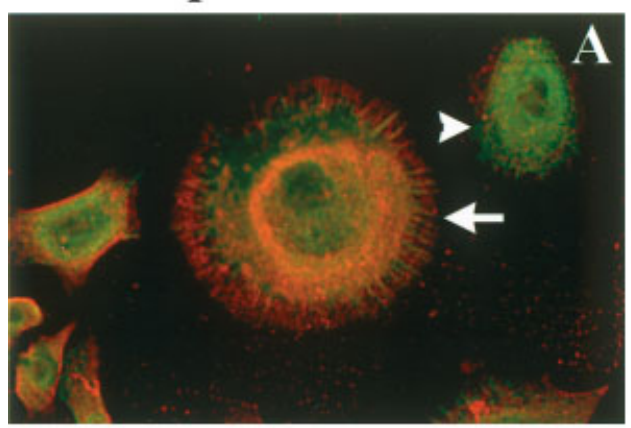

Rap1 + involucrin
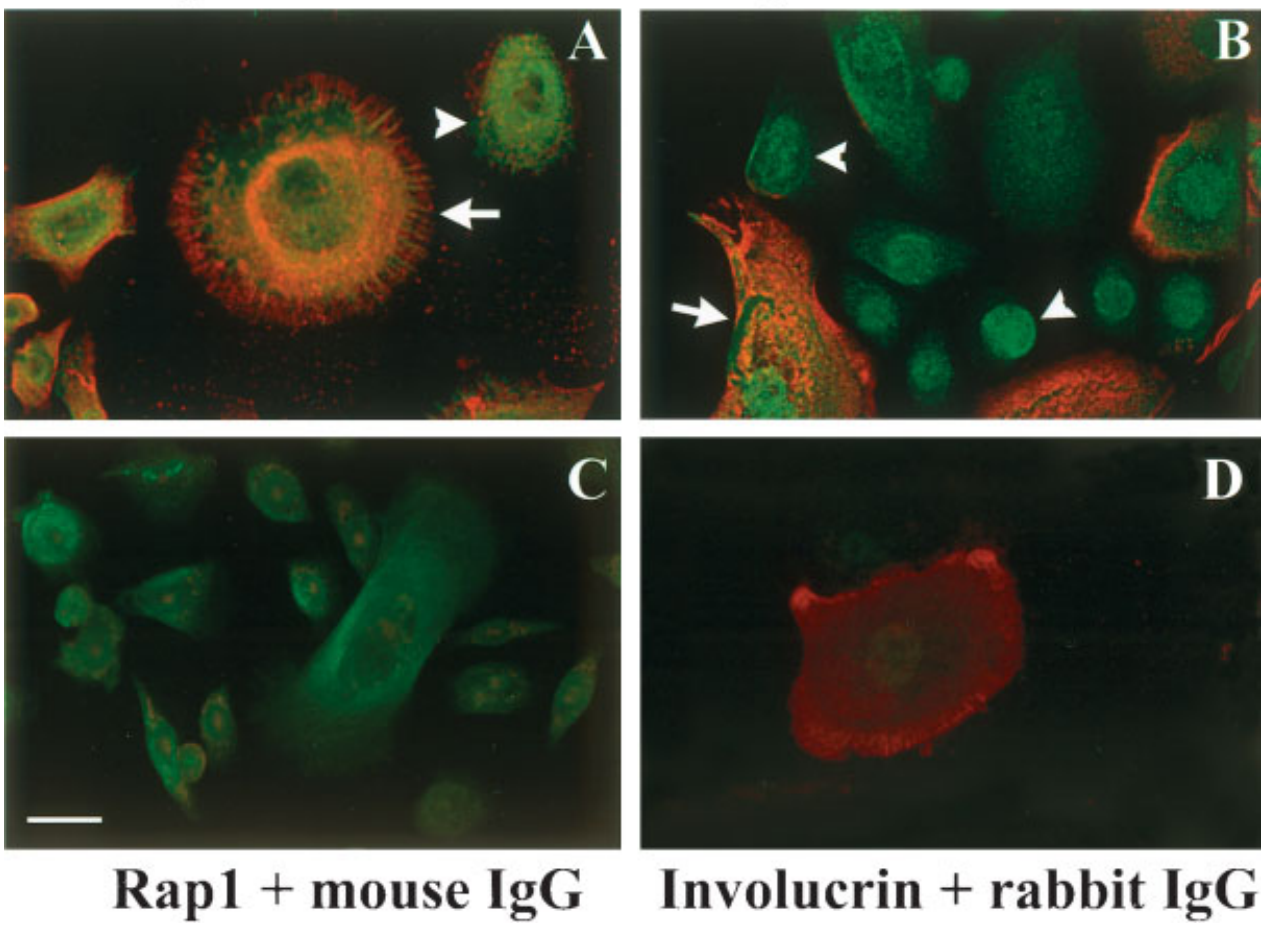

Involucrin + rabbit IgG

Fig. 5. Immunofluorescence detection of rap1 and involucrin in dual labeled immature and mature differentiating primary human oral keratinocytes. P2 HOKs containing both small undifferentiated and large differentiating keratinocytes were cultured on tissue culture slides. The cells were fixed and dual labeled with rabbit anti-rap1 $(\mathbf{A}, \mathbf{B}, \mathbf{C})$ or rabbit $\operatorname{IgG}(\mathbf{D})$ and mouse anti-involucrin $(A, B, D)$ or mouse IgG (C). Immunofluorescence detection was performed as described in "Methods and Materials" $(\mathrm{Bar}=25 \mu \mathrm{m})$. 


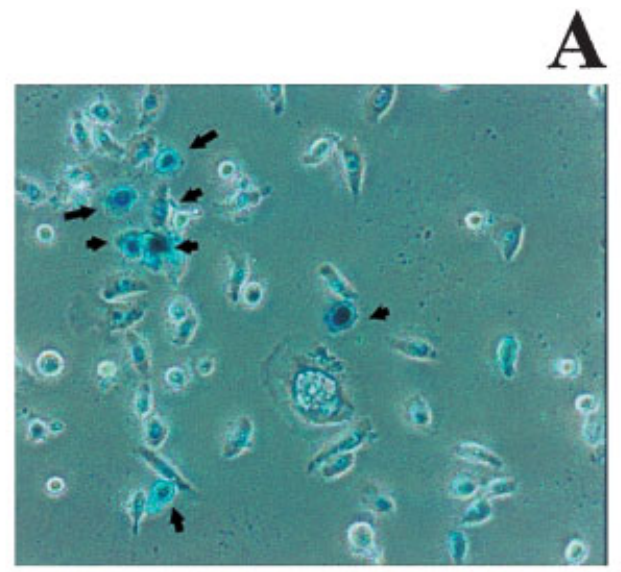

B
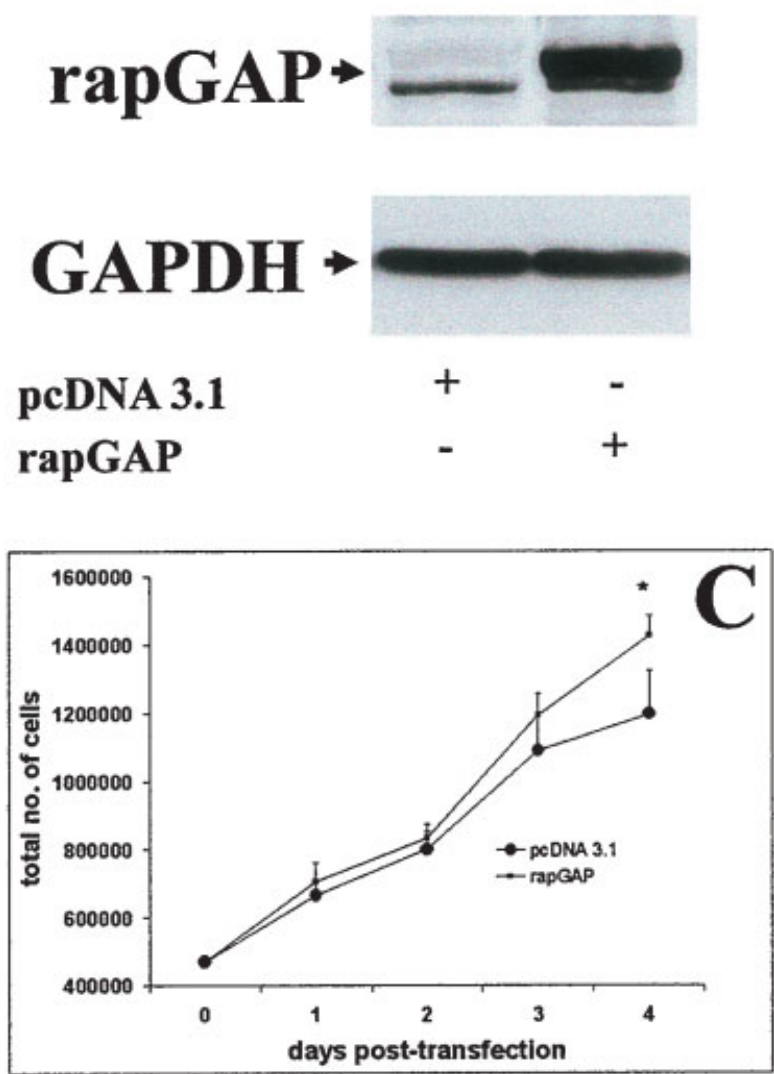

Fig. 6. Proliferation of immortalized keratinocytes transfected with rapGAP. To evaluate transfection efficiency, immortalized human oral keratinocytes were transfected with pcDNA 3.1- $\beta$-gal and evaluated $24 \mathrm{~h}$ later for $\beta$-galactosidase expression $(\mathbf{A})$. Using identical conditions, immortalized keratinocytes were transfected with pcDNA 3.1 -FLAG-rapGAP and control vector. Lysates were electrophoresed, transferred, and exposed to the FLAG antibody to confirm rapGAP expression (B). Proliferation of rapGAP or control vector transfected keratinocytes was determined by a trypan blue enumeration assay (C) as described in the "Materials and Methods." The values represent means of cell number \pm SD based on 2 -wells/data point in a single experiment. *Statistically significant data $(P<0.005$; one-tailed Student's $t$-test). The experiment is representative of three experiments with similar results.
(pcDNA 3.1) transfected cells. In contrast, there was no significant difference in cell viability between cells transfected with control vector or rapGAP, consistent with an increase in cell number due to enhanced proliferation with pcDNA-rapGAP rather than decreased apoptosis in rapGAP transfected cells. Thus, active, GTP-bound rap1 inhibits proliferation in keratinocytes.

\section{DISCUSSION}

In this report, the role of rap1 in differentiation and proliferation in keratinocytes was examined. The major findings of this study were that active GTP-bound rap1 inhibits proliferation, rap1 is upregulated with terminal differentiation in primary oral keratinocytes, and that in this process rap1B mRNA is more strongly expressed than rap1A mRNA. From these observations it was hypothesized, and subsequently shown that exogenously expressed rapGAP, a protein that inactivates both isoforms of rap1, enhances keratinocyte proliferation. Therefore, rap1 may have an anti-proliferative role in oral epithelium.

Rap1A and rap1B, the two rap1 isoforms have been linked to a variety of biological functions, that appear to vary by cell type. Interestingly, the same isoform has been shown to have both tumorigenic and anti-tumorigenic functions (Kitayama et al., 1989; Yoshida et al., 1992). Several studies have been done in mesenchymal cells, thus little is known about the function of the rap1 isoforms in epithelial cells. Since $>80 \%$ of human tumors are epithelial in origin, it is important to characterize the role of these proteins in epithelial cells. Human oral epithelium is a valuable model for studying biochemical and molecular events that regulate or accompany growth and differentiation. Although rap1 and other SMGs have been previously identified in epidermal keratinocytes (Gromov and Celis, 1994), little is known about the expression, regulation and function of SMGs, other than ras isoforms, in epidermal or oral keratinocytes.

Recently, Schmidt et al. (2000) suggested that a SMG, other than ras, regulates differentiation in epidermal keratinocytes via a Raf/Mek/Erk pathway. Earlier, rap1B was shown to play a role in differentiation in neuronal cells (Vossler et al., 1997; York et al., 1998). These studies showed that in PC12 cells, rap1B promotes differentiation by acting via the MAPK pathway (Vossler et al., 1997; York et al., 1998). Subsequently, Schmitt and Stork (2001) showed that cAMP inhibits proliferation in NIH 3T3 cells by acting via rap1. Hence, our initial studies focused on the role of rap1 in terminal differentiation in primary oral keratinocytes.

Keratinocytes undergoing terminal differentiation cease to proliferate, and express biochemical markers of terminal differentiation, such as involucrin, keratins 1 and 10 and filaggrin (Dotto, 1999). In vitro, attached keratinocytes can be induced to differentiate by serial passaging or by treatment with $1.2 \mathrm{mM} \mathrm{CaCl}$ (Norsgaard et al., 1996; Dotto, 1999). However, Norsgaard et al. (1996) reported that differentiation in serially passaged epidermal keratinocytes did not occur spontaneously but rather had to be induced by high calcium concentrations. According to this study, in vitro, keratinocytes senesce or cease to proliferate, but do 
not necessarily differentiate, i.e., express biochemical markers of terminal differentiation. However, in the present study, differentiation, as monitored by increased expression of involucrin, and the loss of proliferative capacity, occurred with sequential passaging. This disparity between the studies may be related to differences in culture conditions or to inherent differences between epidermal and oral keratinocytes. Our studies showed that rap1 expression increases with sequential passages of primary keratinocytes.

Due to the large amount of protein required for rap1 activation assays, we were unable to assess rap1 activation in sequentially passaged primary keratinocytes, which have an inadequate yield for the first and last passages. Hence, we validated and used another in vitro model for terminal differentiation, treating primary keratinocytes with $1.2 \mathrm{mM} \mathrm{CaCl}_{2}$ for 7 days (Norsgaard et al., 1996). In primary keratinocytes treated with high concentrations $(1.2 \mathrm{mM})$ of $\mathrm{CaCl}_{2}$, increased rap1 activation was observed only upon terminal differentiation, i.e., upon cessation of proliferation in addition to involucrin upregulation. An irreversible commitment to differentiation takes more than 6 days of culture under high (1.2 mM) $\mathrm{CaCl}_{2}$ conditions (Norsgaard et al., 1996). Under conditions reported here, $1.2 \mathrm{mM} \mathrm{CaCl}_{2}$ induced proliferation between 1 and 3 days. Expression of active, GTP-bound rap1 increased over the corresponding low calcium control at 7 days, by which time proliferation had ceased. Upregulation of active GTP-bound rap1 was strongly correlated with inhibition of proliferation and was observed at day 7 , even under low calcium conditions probably because of contact inhibition in a confluent plate. Thus, rap1-GTP was upregulated concomitantly with growth cessation and differentiation in both low and high calcium treated samples, but the latter exhibited more prominent effects.

In contrast, active rap1-GTP expression was not upregulated in immortalized keratinocytes upon treatment with $1.2 \mathrm{mM} \mathrm{CaCl}_{2}$ for 7 days. Although these cells expressed the differentiation marker, involucrin, continued proliferation was observed. Altogether, these data suggest that rap1 activation is linked to growth inhibition. Hence, though ras mutations are infrequently identified in oral cancer, our findings suggest that an inactivating mutation in rap1, a protein that antagonizes ras activity (Pessin and Okada, 1999), would lead to uncontrolled proliferation of oral keratinocytes.

The quantitative RT-PCR data suggest that neither rap1 isoform is transcriptionally upregulated during growth cessation, suggesting that the regulatory effect is at the translational or catabolic stage. SMGs regulate biochemical events by shuttling between membranebound and soluble forms depending on whether these proteins are in the active, GTP-bound or inactive, GDPbound state. In fact, we have previously shown that rap1 plays a role in secretion in rat parotid acini by translocating to the cytosolic fraction (Huber et al., 1994). In the present study, the data suggest that the increase in rap1 expression in the soluble fraction of sequential passages of primary keratinocytes, is due to translocation to the soluble fraction or due to effects on protein translation or catabolism. Future studies will explore these possibilities.
In summary, our data show that active, GTP-bound rap1 inhibits proliferation in oral keratinocytes and therefore, is a candidate protein for an anti-tumorigenic role in oral epithelium.

\section{ACKNOWLEDGMENTS}

The authors thank Dr. Cun-Yu Wang, University of Michigan, for helpful comments.

\section{LITERATURE CITED}

Altschuler DL, Ribeiro-Neto F. 1998. Mitogenic and oncogenic properties of the small G protein Rap1b. Proc Natl Acad Sci USA 95:7475-7479.

Altschuler DL, Peterson SN, Ostrowski MC, Lapetina EG. 1995 Cyclic AMP-dependent activation of Rap1b. J Biol Chem 270: 10373-10376.

Barbacid M. 1987. ras Genes. Annu Rev Biochem 56:779-827.

Bokoch GM. 1993. Biology of the Rap proteins, members of the ras superfamily of GTP-binding proteins. Biochem J 289:17-24.

Clark LJ, Edington K, Swan IR, McLay KA, Newlands WJ, Wills LC, Young HA, Johnston PW, Mitchell R, Robertson G, et al. 1993. The absence of Harvey ras mutations during development and progression of squamous cell carcinomas of the head and neck. $\mathrm{Br} \mathrm{J}$ Cancer 68:617-620.

D'Silva NJ, DiJulio DH, Belton CM, Jacobson KL, Watson EL. 1997. Immunolocalization of rap1 in the rat parotid gland: Detection on secretory granule membranes. J Histochem Cytochem 45:965-973.

Dotto GP. 1999. Signal transduction pathways controlling the switch between keratinocyte growth and differentiation. Crit Rev Oral Biol Med 10:442-457.

Farrell F, Torti M, Lapetina EG. 1992. Rap proteins: Investigating their role in cell function. J Lab Clin Med 120:533-537.

Franke B, Akkerman JW, Bos JL. 1997. Rapid $\mathrm{Ca}^{2+}$-mediated activation of Rap1 in human platelets. Embo J 16:252-259.

Gromov PS, Celis JE. 1994. Several small GTP-binding proteins are strongly down-regulated in simian virus 40 (SV40) transformed human keratinocytes and may be required for the maintenance of the normal phenotype. Electrophoresis 15:474-481.

Grunicke HH, Maly K. 1993. Role of GTPases and GTPase regulatory proteins in oncogenesis. Crit Rev Oncog 4:389-402.

Heid CA, Stevens J, Livak KJ, Williams PM. 1996. Real time quantitative PCR. Genome Res 6:986-994.

Herrmann C, Nassar N. 1996. Ras and its effectors. Prog Biophys Mol Biol 66:1-41.

Huber LA, Ullirich O, Takai Y, Lutcke A, Dupree P, Olkkonen V Virta H, de Hoop MJ, Alexandrov K, Peter M, et al. 1994. Mapping of Ras-related GTP-binding proteins by GTP overlay following two-dimensional gel electrophoresis. Proc Natl Acad Sci USA 91: 7874-7878

Jordan RC, Daley T. 1997. Oral squamous cell carcinoma: New insights. J Can Dent Assoc 63:517-518, 521-525.

Kitayama H, Sugimoto Y, Matsuzaki T, Ikawa Y, Noda M. 1989. A ras-related gene with transformation suppressor activity. Cell 56:77-84.

Noda M. 1993. Structures and functions of the K rev-1 transformation suppressor gene and its relatives. Biochim Biophys Acta 1155:97109.

Norsgaard H, Clark BF, Rattan SI. 1996. Distinction between differentiation and senescence and the absence of increased apoptosis in human keratinocytes undergoing cellular aging in vitro. Exp Gerontol 31:563-570.

Oda D, Watson E. 1990. Human oral epithelial cell culture I. Improved conditions for reproducible culture in serum-free medium. In Vitro Cell Dev Biol 26:589-595.

Park NH, Min BM, Li SL, Huang MZ, Cherick HM, Doniger J. 1991. Immortalization of normal human oral keratinocytes with type 16 human papillomavirus. Carcinogenesis 12:1627-1631.

Pessin JE, Okada S. 1999. Insulin and EGF receptors integrate the Ras and Rap signaling pathways. Endocr J 46(Suppl):S11-S16.

Saranath D, Chang SE, Bhoite LT, Panchal RG, Kerr IB, Mehta AR, Johnson NW, Deo MG. 1991. High frequency mutation in codons 12 and 61 of H-ras oncogene in chewing tobacco-related human oral carcinoma in India. Br J Cancer 63:573-578.

Schmidt M, Goebeler M, Posern G, Feller SM, Seitz CS, Brocker EB, Rapp UR, Ludwig S. 2000. Ras-independent activation of the Raf/ MEK/ERK pathway upon calcium-induced differentiation of keratinocytes. J Biol Chem 275:41011-41017. 
Schmitt JM, Stork PJ. 2001. Cyclic AMP-mediated inhibition of cell growth requires the small G protein Rap1. Mol Cell Biol 21:36713683

Vojtek AB, Der CJ. 1998. Increasing complexity of the Ras signaling pathway. J Biol Chem 273:19925-19928.

Vossler MR, Yao H, York RD, Pan MG, Rim CS, Stork PJ. 1997. cAMP activates MAP kinase and Elk-1 through a B-Raf- and Rap1dependent pathway. Cell 89:73-82.

Wagner AC, Williams JA. 1994. Low molecular weight GTP-binding proteins: Molecular switches regulating diverse cellular functions. Am J Physiol 266:G1-G14.
York RD, Yao H, Dillon T, Ellig CL, Eckert SP, McCleskey EW, Stork PJ. 1998. Rap1 mediates sustained MAP kinase activation induced by nerve growth factor [see comments]. Nature 392:622-626.

Yoshida Y, Kawata M, Miura Y, Musha T, Sasaki T, Kikuchi A, Taka Y. 1992. Microinjection of smg/rap1/Krev-1 p21 into Swiss 3T3 cells induces DNA synthesis and morphological changes. Mol Cell Biol 12:3407-3414

Zellmer S, Gaunitz F, Salvetter J, Surovoy A, Reissig D, Gebhardt R 2001. Long-term expression of foreign genes in normal human epidermal keratinocytes after transfection with lipid/DNA complexes. Histochem Cell Biol 115:41-47. 